\title{
Searching for confining hidden valleys at LHCb, ATLAS, and CMS
}

\author{
Aaron Pierce, ${ }^{1}$ Bibhushan Shakya, ${ }^{2,1}$ Yuhsin Tsai, ${ }^{3}$ and Yue Zhao ${ }^{4,1}$ \\ ${ }^{1}$ Leinweber Center for Theoretical Physics, University of Michigan, Ann Arbor, Michigan 48109, USA \\ ${ }^{2}$ Department of Physics, University of Cincinnati, Cincinnati, Ohio 45221, USA \\ ${ }^{3}$ Maryland Center for Fundamental Physics, Department of Physics, University of Maryland, \\ College Park, Maryland 20742, USA \\ ${ }^{4}$ Tsung-Dao Lee Institute, and Department of Physics and Astronomy, Shanghai Jiao Tong University, \\ Shanghai 200240, China
}

(Received 28 August 2017; revised manuscript received 6 November 2017; published 25 May 2018)

\begin{abstract}
We explore strategies for probing hidden valley scenarios exhibiting confinement. Such scenarios lead to a moderate multiplicity of light hidden hadrons for generic showering and hadronization similar to QCD. Their decays are typically soft and displaced, making them challenging to probe with traditional LHC searches. We show that the low trigger requirements and excellent track and vertex reconstruction at $\mathrm{LHCb}$ provide a favorable environment to search for such signals. We propose novel search strategies in both muonic and hadronic channels. We also study existing ATLAS and CMS searches and compare them with our proposals at $\mathrm{LHCb}$. We find that the reach at $\mathrm{LHCb}$ is generically better in the parameter space we consider here, even with optimistic background estimations for ATLAS and CMS searches. We discuss potential modifications at ATLAS and CMS that might make these experiments competitive with the LHCb reach. Our proposed searches can be applied to general hidden valley models as well as exotic Higgs boson decays, such as in twin Higgs models.
\end{abstract}

DOI: 10.1103/PhysRevD.97.095033

\section{INTRODUCTION}

To ensure no physics remains hidden, experiments at the LHC must search for a wide variety of phenomena beyond the Standard Model (SM). Most searches focus on signatures with energetic particles from a primary vertex or with large missing energy. However, several well-motivated scenarios possess signatures that are challenging for such traditional LHC searches, motivating novel search strategies. We study such strategies for hidden valley (HV) scenarios [1], which produce multiple soft and displaced objects.

HV scenarios consist of a sector with light (e.g., GeV scale) particles connected to the SM sector only via massive particles, effectively forming a barrier between the sectors. The hidden sector may be confined via a non-Abelian gauge symmetry, with a model-dependent mass spectrum. An approximate chiral symmetry spontaneously broken by hidden sector confinement leads to light hidden pions, $\pi_{v}$; otherwise, the lightest particles may be hadrons with masses comparable to or heavier than the hidden confinement scale $\Lambda_{v}$, as in, e.g., fraternal twin Higgs models [2].

Published by the American Physical Society under the terms of the Creative Commons Attribution 4.0 International license. Further distribution of this work must maintain attribution to the author(s) and the published article's title, journal citation, and DOI. Funded by SCOAP .
Showering and hadronization (SH) in the hidden sector produces many hadrons, each much softer than the total energy. Hadron multiplicity can range from a few in the absence of light quarks to $\mathcal{O}(1000)$ [3] for a long showering window with large 't Hooft coupling. We focus on theories averaging $\mathcal{O}(10)$ hadrons per event, as in QCD, and hadron lifetimes long enough for displaced vertices (DV) at the LHC. Complementary strategies that instead use kinematic information in such dark showers are discussed in Refs. [4,5].

At $\mathrm{LHCb}$, planned upgrades will remove hardware-level triggers entirely, dramatically improving search flexibility. Furthermore, excellent vertex reconstruction, invariant mass resolution, particle identification, and a lower pileup environment make LHCb well suited for exotic soft longlived particle searches. Previous HV searches at LHCb $[6,7]$ focused on final states with only two hard $\pi_{v}$ and are not optimal for larger multiplicity. Our proposed searches instead have similarities with existing LHCb SM searches for $K_{S} \rightarrow \mu^{ \pm}$[8] and $B_{0} \rightarrow D^{ \pm}$[9].

Although stringent trigger requirements and potentially significant backgrounds make HV searches at ATLAS and CMS challenging, these experiments enjoy $\sim 20 \times$ the luminosity and $\sim 10 \times$ the angular coverage of LHCb. Specialized triggers for displaced decays exist (e.g., Refs. [10-14]) but are limited in efficiency and scope. We study the efficacy of various existing ATLAS and CMS searches and mention possible modifications for improvement. 


\section{SETUP}

We consider a heavy $U(1)^{\prime}$ gauge boson $Z_{p}$ with gauge coupling $g_{v}$, coupled to both SM quarks $q$ and HV quarks $q_{v}$ with charges $Q_{v}^{\mathrm{SM}}, Q_{v}^{H V}$ :

$$
L \supset g_{v} Z_{p}^{\mu}\left(Q_{v}^{\mathrm{SM}} \bar{q} \gamma_{\mu} q+Q_{v}^{H V} \bar{q}_{v} \gamma_{\mu} q_{v}\right)+\frac{1}{2} m_{Z_{p}}^{2} Z_{p}^{2} .
$$

If $Q_{v}^{\mathrm{SM}} \ll Q_{v}^{H V}$, as occurs when the $\mathrm{SM}$ coupling arises from kinetic mixing [15], the $Z_{p}$ primarily decays to the hidden sector; this alleviates bounds from direct $Z_{p}$ searches [16]. For concreteness, we assume the low energy HV spectrum possesses two approximately mass degenerate hadrons: a composite vector boson $\omega_{v}$ and a pseudoscalar $\eta_{v}$ [1], which decay into SM states. The $\omega_{v}$ lifetime is model dependent and is treated a free parameter. Chiral suppression ensures a longer $\eta_{v}$ lifetime-we assume they escape. ${ }^{1}$ The process of interest is

$$
\begin{aligned}
q \bar{q} & \rightarrow Z_{p} \rightarrow q_{v} \bar{q}_{v} \rightarrow N_{\omega_{v}} \times \omega_{v}+N_{\eta_{v}} \times \eta_{v}, \\
\omega_{v} & \rightarrow f \bar{f}(\text { displaced decay }) .
\end{aligned}
$$

We assume $3 / 4$ of the light mesons are $\omega_{v}$, due to the spin degrees of freedom. We consider $\omega_{v}$ masses from $\mathcal{O}(100) \mathrm{MeV}$ to a few $\mathrm{GeV}$, comparable to the mass scale of SM hadrons. The choice of lifetimes corresponding to decay lengths $\lesssim 1 \mathrm{~m}$, in additional to being favorable for $\mathrm{LHCb}$ searches, is also motivated by constraints from existing dark photon searches and cosmology, where this regime is preferred to satisfy energy density constraints from big bang nucleosynthesis [17].

Exotic decays of the Higgs boson to HV particles $[7,18]$ give similar final states:

$$
g g \rightarrow h \rightarrow q_{v} \bar{q}_{v} \rightarrow N_{\omega_{v}} \times \omega_{v}+N_{\eta_{v}} \times \eta_{v} .
$$

We will also study this process in a variation of the twin Higgs (TH) model.

SH in the hidden sector govern the average number and kinematics of the hidden hadrons. We implement SH using a modified version of the PYTHIA8.1 HV implementation [19], as described in Ref. [20]. ${ }^{2}$ We compare with results from a simplified analytical procedure using the quark-combination model (QCM) [22-25] with the longitudinal phase space approximation (LPSA) [26,27]. While different approaches give different average multiplicity $\left\langle N_{v}\right\rangle \equiv\left\langle N_{\omega_{v}+\eta_{v}}\right\rangle$, once $\left\langle N_{v}\right\rangle$ is fixed, we find similar kinematic distributions. Thus, we treat $\left\langle N_{v}\right\rangle$, which depends on details of the gauge group, such as the confinement scale, as a free parameter. We discuss several

\footnotetext{
${ }^{1}$ For shorter $\omega_{v}$ lifetimes, the signals discussed here might instead be applicable to $\eta_{v}$ decays.

${ }^{2}$ This gives results consistent with the latest PYTHIA version [21], which incorporates running effects.
}

search strategies for hidden hadron decay to (i) muon pairs and (ii) heavy quarks, at both LHCb and ATLAS and CMS.

\section{DIMUON CHANNEL}

\section{A. LHCb strategies}

Displaced muon-pair searches at LHCb are discussed in Refs. [28,29]. The dark photon search strategy of Ref. [29] is directly applicable to our scenario. We apply the following selection criteria:

(i) $\geq 1 \mathrm{DV}$ with transverse displacement $\ell_{T} \in[6,22] \mathrm{mm}$.

(ii) Pseudorapidity $\eta\left(\omega_{v}\right) \in[2,5]$; each vertex contains two opposite-sign muons with $\eta\left(\mu^{ \pm}\right) \in[2,5]$.

(iii) $p_{T}\left(\mu^{ \pm}\right)>0.5 \mathrm{GeV}, p\left(\mu^{ \pm}\right)>10 \mathrm{GeV}$.

These ensure the DV is well separated from the beam line and the two muons can be reconstructed as tracks in the vertex locator (VELO) with muon identification efficiency $\varepsilon_{\mu}^{2} \approx 0.5$. The transverse displacement and $\eta$ requirements effectively impose a cut on the longitudinal displacement $\lesssim 1 \mathrm{~m}$, forcing the decay within the VELO.

Figure 1 (left panel) presents the LHCb sensitivity to the $Z_{p}$ model with $15 \mathrm{fb}^{-1}$ of data. The width of the bands represents the variation $10 \leq\left\langle N_{v}\right\rangle \leq 30$. We adopt the background $\approx 25$ events per mass bin from Ref. [29], where the mass bin is twice the $m_{\mu \mu}$ resolution. Results are in red $\left(m_{\omega_{v}}=0.3 \mathrm{GeV}\right)$ and blue $\left(m_{\omega_{v}}=0.6 \mathrm{GeV}\right)$. PythiA and the QCM + LPSA method are compared to illustrate the scheme independence of our results. We display the reach requiring two DV, assuming zero background. We show the $Z_{p}$ production cross section with a photonlike coupling suppressed by $\epsilon=0.02$.

Figure 1 (right panel) shows analogous results for exotic Higgs decay as brown $\left(m_{\omega_{v}}=6 \mathrm{GeV}\right)$ and orange $\left(m_{\omega_{v}}=2 \mathrm{GeV}\right)$ bands. We also explore an incarnation of the fraternal TH model [2,30], with $\hat{\Lambda}_{\mathrm{QCD}}<m_{\hat{c}} \simeq m_{\hat{s}}<m_{\hat{A}}<$ $m_{\hat{b}} \simeq m_{\hat{l}, \hat{\nu}}<m_{\hat{t}}$ in the twin sector. We focus on $g g \rightarrow$ $h \rightarrow \hat{b} \bar{b}^{3}$, with subsequent decays $\hat{b} \rightarrow \hat{c} \overline{\hat{c}} \hat{s}$. This eventually produces $\hat{\omega} / \hat{\eta}$, comprised of the light flavor, which can decay as $\hat{\omega} \rightarrow \mu^{+} \mu^{-}$through a kinetic mixing $\epsilon$ between the SM and twin photons. ${ }^{4}$ Although the light twin mesons are primarily produced by heavy twin $B$ meson decays (rather than $\mathrm{SH}$ ), the multiplicity is low, and the kinematic distributions are similar to the $\mathrm{SH}$ case, giving a comparable reach (green curve). The prediction in the TH scenario (green line) ${ }^{5}$ demonstrates LHCb's ability to probe such models for $c \tau_{\hat{\omega}} \lesssim 1 \mathrm{~m}$, where $c \tau_{\hat{\omega}} \simeq 0.1 \mathrm{~m}\left(\frac{\mathrm{GeV}}{m_{\hat{c}, \hat{s}}}\right)^{3}\left(\frac{m_{\hat{A}}}{20 \mathrm{GeV}}\right)^{4}\left(\frac{10^{-3}}{\epsilon}\right)^{2}\left(\frac{\mathrm{GeV}}{\hat{\Lambda}_{\mathrm{QCD}}}\right)^{2}$ [17]. The signal is

\footnotetext{
${ }^{3}$ We ignore comparable decays into twin leptons, which can be invisible or produce similar signals via the $\tau^{\prime} \rightarrow \nu^{\prime}+$ hidden hadron.

${ }^{4}$ We assume the $U(1)^{\prime}$ is broken, and no symmetry prevents the decay of mesons like $\bar{c} s$.

${ }^{5}$ We set the ratio between the twin sector and SM vacuum expectation values as $f / v=4$, consistent with existing constraints and modest fine-tuning.
} 

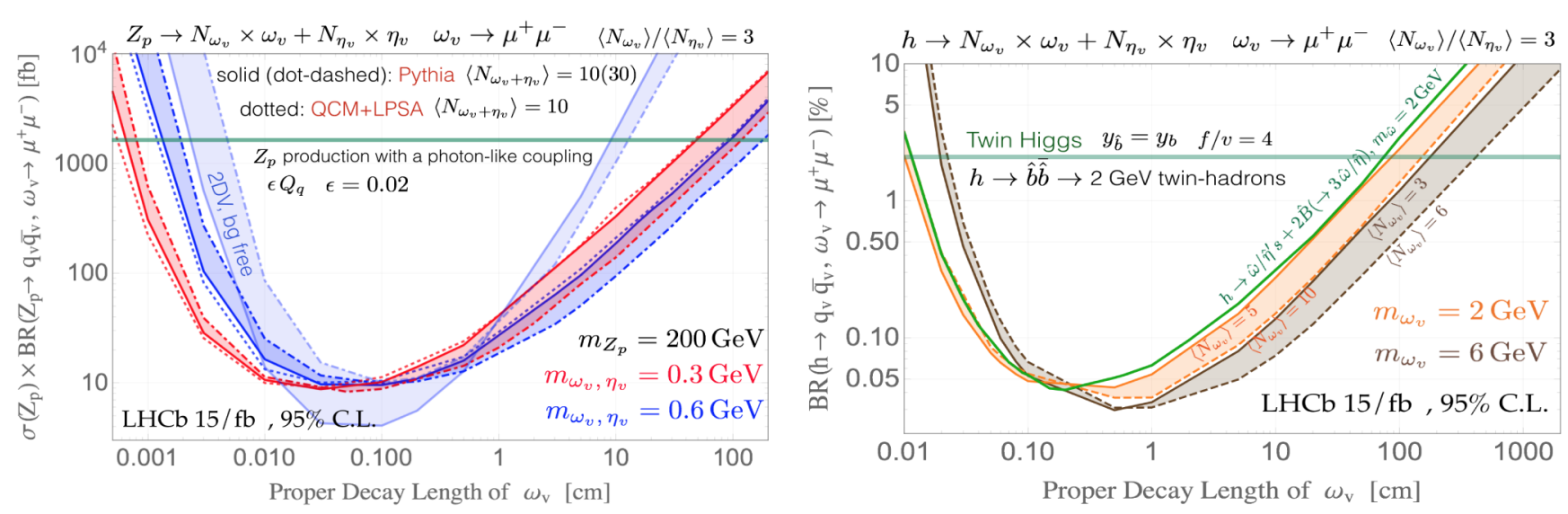

FIG. 1. Left: $Z_{p}$ cross section reach. Green line: cross section for a photonlike coupling, suppressed by $\epsilon=0.02$. Right: Projected upper bounds on $\operatorname{BR}(h \rightarrow$ twin bottom quarks $)$ using the one DV search. This process produces $\hat{\omega} / \hat{\eta}$ followed by $\hat{\omega} \rightarrow \mu^{+} \mu^{-}$. Horizontal green line: prediction in a variation of the fraternal TH model; $\omega_{v}$ is a mixture of $c^{\prime}$ and $s^{\prime}$. Green curve: reach for the corresponding decay topology. The width of the bands correspond to $10 \leq\left\langle N_{v}\right\rangle \leq 30$.

sensitive to the twin sector spectrum; we postpone studies of variations to future work.

The produced $\omega_{v}$ are highly boosted, but the precise value of the boost depends on the $\omega_{v}$ mass. For decay lengths $\gtrsim 1 \mathrm{~cm}$, the bounds weaken with increasing lifetimedecays become less likely to occur in the detector. In this case, a heavier $\omega_{v}$ is less boosted and has a higher probability of decaying inside the detector. Below $\lesssim \mathcal{O}(0.1) \mathrm{cm}$, the postmodule search strategy is not opti$\mathrm{mal}$, and hence the bounds deteriorate. Lighter $m_{\omega_{v}}$ results in larger boosts; with longer lifetime, the decay probability in the signal region, i.e., $\ell_{T} \geq 6 \mathrm{~mm}$, is larger. These factors, which affect the efficiency for decays to occur with the proper transverse displacement, explain the relative placement of the curves corresponding to different $\omega_{v}$ masses.

For the $Z_{p}$ case with $c \tau_{\omega_{v}} \gtrsim 10 \mathrm{~cm}$, the acceptance of an individual meson changes by $\approx 50 \%$ when $\left\langle N_{v}\right\rangle$ changes from 10 to 30 ; thus, the reach is approximately proportional to $\left\langle N_{v}\right\rangle$. This can be understood from the softness of the showering process: the distribution of hadrons from $\mathrm{SH}$ peaks at low boost, and this part of the distribution is relatively insensitive to $\left\langle N_{v}\right\rangle$. The high boost portion, which is more sensitive to $\left\langle N_{v}\right\rangle$, is removed by the requirement of decaying inside the VELO. For sufficiently short decay lengths, even highly boosted particles decay within the detector. In this case, a smaller $\left\langle N_{v}\right\rangle$ generates hadrons with higher momenta, which helps pass the lower cut on transverse displacement.

\section{B. ATLAS and CMS searches}

We consider three existing triggers. First, multiple displaced soft muons are naively the closest fit to our topology. The other two, $E_{T}$ and a hard displaced muon, give meaningful bounds despite not exactly matching the signal features. Ordinary multilepton searches reject muons with large displacements to remove cosmic-ray muon background [31] and do not apply. Searches for displaced objects relax the cut, necessitating proper treatment of the cosmic-ray background.

\section{Multiple displaced soft muons}

Reference [14] used a trimuon trigger for displaced lepton jet (DLJ) searches, selecting events having $\geq 3$ muon spectrometer (MS)-only tracks with $p_{T}>6 \mathrm{GeV}$ within a $\Delta R<0.4$ cone. This requires at least two hidden hadrons to decay between $4 \mathrm{~m}<\ell_{T}<6.5 \mathrm{~m}$ within $\Delta R<0.4$. The analysis required an additional displaced decay with $\Delta R \geq 0.4$ separation from the ones used for the trigger, with a more inclusive $10 \mathrm{~cm}<\ell_{T}<6.5 \mathrm{~m}$. This necessitates the decay of a third hidden hadron. We require $p_{T}>6 \mathrm{GeV}$ for each muon. For the muon-pair reconstruction efficiency, we adopt the value of 0.4 reported in the analogous CMS displaced muon search [32] for the decay of a $150 \mathrm{GeV}$ particle, although a lower efficiency may be expected for softer muons. To estimate background, which is dominantly from multijet events and cosmic muons, we rescale the background from [14] to $300 \mathrm{fb}^{-1}$, optimistically assuming systematic uncertainties improve as $\sqrt{N}$. The corresponding reach curve (Fig. 2, two DLJs, brown band) is worse than that from LHCb (blue band, corresponding to the red band of Fig. 1). To reduce the background, one may require another displaced vertex with the looser $r$ cut and $\Delta R \geq 0.4$ from the initial DLJs. For proper decay lengths between 1 and $10 \mathrm{~cm}$, such that displaced decays within the detector are likely, this (three DLJs, green band) can be competitive with LHCb assuming negligible background. Requiring too many hidden hadrons to decay within the displaced region hurts signal acceptance. Relaxing this by, e.g., including the inner detector for the trimuon trigger could improve the reach dramatically. 


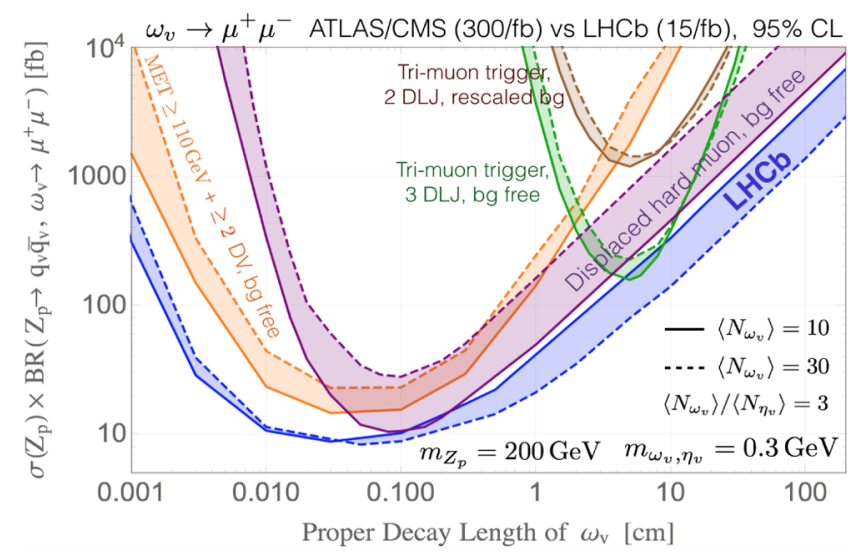

FIG. 2. Projected bounds from various ATLAS and CMS displaced muons search strategies. The brown curve represents an extrapolation of a current ATLAS analysis, while the green curve represents a minor modification. The orange and purple projections have aggressive assumptions about backgrounds and will likely weaken following detailed detector simulations. The blue band is from the LHCb search proposed in this work.

\section{2. $E_{T}$ trigger}

For long lifetimes, most hidden hadrons escape and contribute to missing energy, ${ }^{6}$ particularly if recoiling against a hard object, motivating a $E_{T}+$ (displaced) muons search. An ATLAS $E_{T}+$ DV search [13] triggered on events with $E_{T}$ then further (at the analysis level) requires a hard muon $\left(p_{T}>55 \mathrm{GeV}\right)$. To optimize for our signal, we relax the $E_{T}$ down to the trigger requirement [35]. We then relax the muon $p_{T}$ cut, demanding instead two dimuon $\mathrm{DVs}^{7}$ :

(i) $E_{T} \geq 110 \mathrm{GeV}$.

(ii) Reconstruct $\geq 2 \mathrm{DVs}$, each with $\ell_{T} \in[1,30] \mathrm{cm}$.

(iii) Each muon has $p_{T}>10 \mathrm{GeV}$.

The $\ell_{T}$ requirement ensures they are inside the silicon detector to reconstruct the muon in the tracker (see Fig. 6 in Ref. [10]). With a DV reconstruction efficiency of 0.4 and an optimistic assumption of no background, the reach is shown in Fig. 2 (orange band), which is weaker than our $\mathrm{LHCb}$ projection.

\section{Hard displaced muon}

Following the analysis in Ref. [13], we require:

(i) $\geq 1$ reconstructed DV, each with $\ell_{T} \in[1,30] \mathrm{cm}$.

(ii) $\geq 1$ muon with $p_{T} \geq 55 \mathrm{GeV}$ and transverse impact factor $>1.5 \mathrm{~mm}$ and $p_{T}>10 \mathrm{GeV}$ for the other displaced muon from this vertex.

\footnotetext{
${ }^{6}$ We define $E_{T}$ as the opposite of the vector sum of all wellconstructed objects. We do not include the (displaced) muons in this category. Our $E_{T}^{\prime}$ definition should align with that in Refs. [33,34].

Using multiple displaced vertices was also discussed in Ref. [36].
}

For light hidden hadrons, the DV invariant mass cut imposed in Ref. [13] must be removed. There are already $\mathcal{O}(1)$ background events in the low invariant mass region in this $8 \mathrm{TeV}, 20.3 / \mathrm{fb}$ search (see Fig. 9 in Ref. [13]); the precise number is difficult to estimate since the dominant background is likely combinatoric in nature. Regardless, this search has a slightly weaker projected reach than LHCb even assuming no background (Fig. 2, purple band). We expect sensitivity to significantly improve if the stringent $p_{T}(\mu)$ cut could be relaxed while maintaining low background.

\section{HEAVY FLAVOR DECAY CHANNELS}

\section{A. LHCb strategies}

For decays to $c \bar{c}$, subsequent SM hadronization often produces two $D$ mesons, $D_{(s)}^{0}, \bar{D}_{(s)}^{0}$ or $D^{ \pm}$. The nonnegligible lifetimes of charmed hadrons create an additional separation between the two $D$ meson decay positions, producing two vertices with large separation from the primary vertex and a small but significant separation from each other. Resolving the secondary vertices should be straightforward as the position resolution in the VELO is $\mathcal{O}(10) \mu \mathrm{m}$ while $c \tau_{D} \sim \mathcal{O}(100) \mu \mathrm{m}$. We do not explicitly require a minimum separation between the two vertices, but note this could be implemented to further reject background if necessary. Several strategies to identify $D$ mesons at LHCb exist, some without full reconstruction [37]. Given the sizable probability for one $D$ meson to decay to $\geq 3$ charged tracks that can be well reconstructed in the VELO, we consider the following increasingly inclusive search strategies:

(i) Two nearby reconstructed displaced $D$ mesons (2D).

(ii) One displaced reconstructed $D$ meson and one DV with $\geq 3$ charged tracks nearby (1D1V).

(iii) Two DV, each with $\geq 3$ charged tracks, near each other $(2 \mathrm{~V})$.

When reconstructing a $D$ meson, we focus on channels containing only charged particles: $\operatorname{BR}\left(D^{+} \rightarrow K^{-} 2 \pi^{+}\right) \sim 9.5 \%$, $\operatorname{BR}\left(D^{0} \rightarrow K^{-} 2 \pi^{+} \pi^{-}\right) \sim 8 \%$, and $\operatorname{BR}\left(D_{s}^{+} \rightarrow K^{+} K^{-} \pi^{+}\right) \sim 5 \%$. Meanwhile, requiring $\geq 3$ charged tracks rather than a reconstructed $D$ meson selects the decays $\operatorname{BR}\left(D^{+} \rightarrow 3\right.$-prong $) \sim 18.6 \%, \operatorname{BR}\left(D^{0} \rightarrow 4\right.$-prong $) \sim 14.5 \%$, and $\operatorname{BR}\left(D_{s}^{+} \rightarrow 3-\right.$ prong $) \sim 12 \%$.

A well-defined track must have $p_{T}>0.1 \mathrm{GeV}$ and hit at least three disks in the VELO; with these, we expect good track reconstruction resolution despite the displacement. To retain $\sim 90 \%$ of the signal while rejecting cosmic rays and combinatoric background, we impose varying maximum displacement requirements. For 2D events, the boost factor for each $D$ meson is known; we require $d_{D} \lesssim 5 c \tau_{D} \gamma_{\max }$, where $d_{D}$ is the two $D$ mesons' separation in the laboratory frame and $\gamma_{\max }$ is the larger of the two boost factors. For $1 \mathrm{D} 1 \mathrm{~V}$ events, we require $d_{D}<5 c \tau_{D} \gamma_{D}$ and $d_{T, D}<8 c \tau_{D}$, where $d_{T, D}$ is the transverse distance between the $D$ meson and the DV. Finally, for $2 \mathrm{~V}$ events, we require $d_{T, D}<5 c \tau_{D}$ 


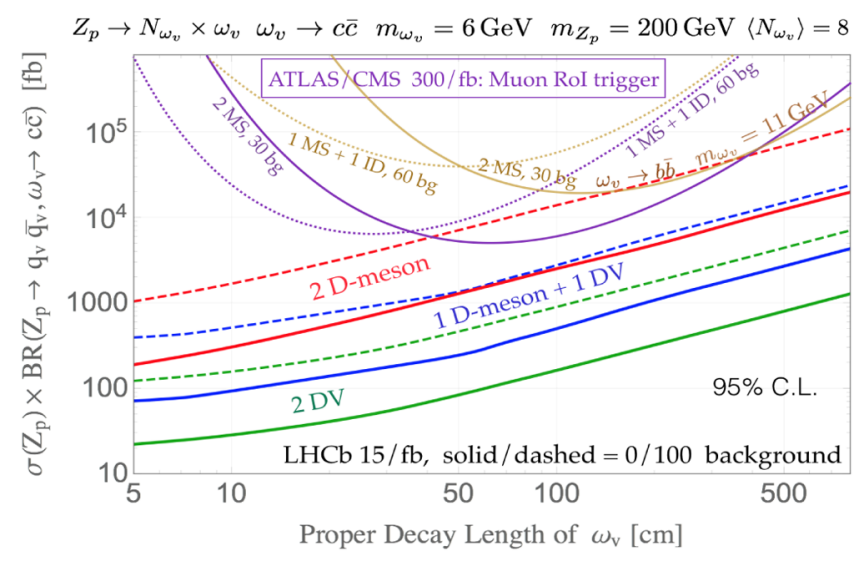

FIG. 3. Projected bounds from various displaced $c \bar{c}$ search strategies. Purple curves: ATLAS and CMS reach estimate for DV decays into $\geq 5$ charged tracks, with either two DV in the muon spectrometer (solid) or one DV in the inner detector and one in the muon spectrometer (dotted). Brown: analogous ATLAS and CMS reach for $\omega_{v} \rightarrow b \bar{b}, m_{\omega_{v}}=11 \mathrm{GeV}$.

and $d_{Z, D}<30 c \tau_{D}$, where $d_{Z, D}$ is the distance between the two vertices along the beam direction. We require the $D$ meson decay $>10 c \tau_{D}$ away from the primary vertex to eliminate SM heavy quark backgrounds. Following the $B^{0} \rightarrow D^{+} D^{-}$search in Ref. [9], when reconstructing a $D$ meson, we require the scalar sum of the charged tracks' $p_{T}$ to exceed $1.8 \mathrm{GeV}$. When identifying a $D$ meson pair, we require the scalar sum of the $p_{T}$ of the two mesons and the total momentum to exceed 5 and $10 \mathrm{GeV}$ respectively.

Note that the displaced vertices in the analysis we propose here are generally more displaced than those in the $B^{0} \rightarrow D^{+} D^{-}$search. Thus, the background could differ from that in Ref. [9]. For instance, such displaced vertices could be generated by interactions with the VELO material. Such background might be mitigated by requiring the reconstructed vertices to be well away from the VELO material. There could also be non-negligible background from combinatorics. Estimates of such backgrounds require a detailed detector simulation. Since this is beyond the scope of this paper, we present our results in Fig. 3 assuming 0,100 background, which requires 3,20 signal events with $15 \mathrm{fb}^{-1}$ data for $2 \sigma$ bounds. While it is not possible to extract a precise background, considering the analysis in Ref. [9], these numbers likely bracket the true value. The limits degrade with increasing lifetime because the decay particles are less likely to leave a sufficient number of hits. The limits also degrade at shorter decay lengths (analogous to Figs. 1 and 2), but we do not plot this region as SM backgrounds become important for displacement $\lesssim \mathcal{O}(\mathrm{cm})$.

\section{B. ATLAS and CMS searches}

An ATLAS search for long-lived hadronic decays [10], targeting $\mathrm{HV} Z_{p}$ and stealth supersymmetry models, applies two different triggers: jet $+E_{T}$ and a muon region of interest (RoI) cluster trigger. Post-trigger, both require $\geq 2 \mathrm{DV}$ at the analysis level. Two types of DVs are considered: an inner detector (ID) vertex, covering $5 \mathrm{~cm}<r<30 \mathrm{~cm}$, or an MS vertex, covering the region between the outer edge of the hadronic calorimeter and the middle station of the muon chambers, $4 \mathrm{~m}<r<6.5 \mathrm{~m}$. The jet $+E_{T}$ trigger requires a leading jet with $p_{T}>$ $120 \mathrm{GeV}, E_{T}>200 \mathrm{GeV}$, and $\geq 7$ charged tracks in each DV. Given the small probability of getting $\geq 7$ tracks in our scenarios (see branching ratio (BR) into three-prong and four-prong topologies listed earlier), this trigger is not optimal.

The muon RoI cluster triggered events require $\geq 5$ charged tracks at each DV, three of which have $p_{T}>$ $10 \mathrm{GeV}$. We estimate bounds for both two MSs displaced decays or one MS and one ID vertex in Fig. 3, rescaling the background in Ref. [10], which mainly comes from jets with high multiplicity tracks, to $300 \mathrm{fb}^{-1}$. While this extension appears weaker than the $\mathrm{LHCb}$ reach, further optimization might yield improvement. For instance, the number of charged tracks required in Ref. [10] was optimized for specific benchmark models and may not be ideal for all scenarios. Requiring more DVs, however, does not appear promising; while this may eliminate background, the signal efficiency is such that the reach worsens.

The above analyses can be repeated without major modifications for the $b \bar{b}$ channel as a $B$ meson decay is very likely to produce a $D$ meson. At LHCb, more detailed $B$ meson reconstruction likely removes more background, leading to comparable or potentially better reach than the $c \bar{c}$ channel. At ATLAS and CMS, a greater number of tracks in $b \bar{b}$ decay favors the $\geq 7$ charged tracks requirement but reduces the $p_{T}$ of each track, resulting in a worse reach than the $c \bar{c}$ channel (Fig. 3, brown curves). The relative sensitivity to the $c \bar{c}$ and $b \bar{b}$ channels depends on $m_{Z_{p}}$ as well as $\left\langle N_{\omega_{v}}\right\rangle$.

\section{CONCLUSIONS}

Our studies indicate that $\mathrm{LHCb}$ is well suited to search for decays of soft long-lived particles in dimuon and heavy flavor channels as realized in confining HV scenarios, with good reach for $Z_{p}$ models and a realization of the TH model. We emphasize that LHCb can also directly search for new electroweak physics rather than rely on indirect flavor signals. Projections from existing ATLAS and CMS strategies (designed for other searches) appear comparatively worse, as even the displaced searches often require energetic particles or large missing energy, which are inefficient for HV searches. Our studies indicate that using additional displaced vertices instead in the trigger or as a background discriminant could dramatically improve sensitivities, potentially making ATLAS and CMS competitive with LHCb (related ideas have recently been discussed in 
Ref. [38]). With such improvements, LHCb and ATLAS and CMS can provide complementary coverage in the search for confining hidden valley scenarios at the LHC.

\section{ACKNOWLEDGMENTS}

We are grateful to David Curtin, Yuanning Gao, Brian Hamilton, Philip Ilten, Hassan Jawahery, Simon Knapen, Daniel Levin, William Parker, Heather Russell, Raman Sundrum, Jesse Thaler, Andreas Weiler, Mike Williams, and Zhenwei Yang for useful discussions. This work was performed in part at the Aspen Center for Physics, which is supported by National Science Foundation Grant No. PHY1066293. A. P. and Y. Z. are supported by U.S. Department of Energy under Grant No. DE-SC0007859. Y. T. is supported by the NSF under Grant No. NSF-PHY1620074 and the Maryland Center for Fundamental Physics. B. S. acknowledges support from the University of Cincinnati and thanks the CERN and DESY theory groups, in which part of this work was conducted, for hospitality. Y.Z. also thank the support of grant from the Office of Science and Technology, Shanghai Municipal Government (Grant No. 16DZ2260200).
[1] M. J. Strassler and K. M. Zurek, Phys. Lett. B 651, 374 (2007).

[2] N. Craig, A. Katz, M. Strassler, and R. Sundrum, J. High Energy Phys. 07 (2015) 105.

[3] S. Knapen, S. Pagan Griso, M. Papucci, and D. J. Robinson, J. High Energy Phys. 08 (2017) 076.

[4] T. Cohen, M. Lisanti, H. K. Lou, and S. Mishra-Sharma, J. High Energy Phys. 11 (2017) 196.

[5] T. Cohen, M. Lisanti, and H. K. Lou, Phys. Rev. Lett. 115, 171804 (2015).

[6] R. Aaij et al. (LHCb Collaboration), Eur. Phys. J. C 75, 595 (2015).

[7] R. Aaij et al. (LHCb Collaboration), Eur. Phys. J. C 76, 664 (2016).

[8] R. Aaij et al. (LHCb Collaboration), Eur. Phys. J. C 77, 678 (2017).

[9] R. Aaij et al. (LHCb Collaboration), Phys. Rev. Lett. 117, 261801 (2016).

[10] G. Aad et al. (ATLAS Collaboration), Phys. Rev. D 92, 012010 (2015).

[11] The ATLAS collaboration, Tech. Rep. ATLAS-CONF2016-103, CERN, Geneva, 2016, http://cds.cern.ch/ record/2219571.

[12] V. Khachatryan et al. (CMS Collaboration), Phys. Rev. D 91, 012007 (2015).

[13] G. Aad et al. (ATLAS Collaboration), Phys. Rev. D 92, 072004 (2015).

[14] The ATLAS collaboration, Tech. Rep. ATLAS-CONF2016-042, CERN, Geneva, 2016, http://cds.cern.ch/ record/2206083.

[15] B. Holdom, Phys. Lett. 166B, 196 (1986).

[16] D. Curtin, R. Essig, S. Gori, and J. Shelton, J. High Energy Phys. 02 (2015) 157.

[17] H.-C. Cheng, S. Jung, E. Salvioni, and Y. Tsai, J. High Energy Phys. 03 (2016) 074.

[18] M. J. Strassler and K. M. Zurek, Phys. Lett. B 661, 263 (2008).

[19] T. Sjostrand, S. Mrenna, and P. Z. Skands, Comput. Phys. Commun. 178, 852 (2008).

[20] P. Schwaller, D. Stolarski, and A. Weiler, J. High Energy Phys. 05 (2015) 059.
[21] T. Sjöstrand, S. Ask, J. R. Christiansen, R. Corke, N. Desai, P. Ilten, S. Mrenna, S. Prestel, C. O. Rasmussen, and P. Z. Skands, Comput. Phys. Commun. 191, 159 (2015).

[22] Q.-B. Xie and X.-M. Liu, Phys. Rev. D 38, 2169 (1988).

[23] Q. Wang, Z.-G. Si, and Q.-B. Xie, Int. J. Mod. Phys. A 11, 5203 (1996).

[24] Z.-G. Si, Q.-B. Xie, and Q. Wang, in Proceedings, 7th International Workshop on Correlations and Fluctuations in Multiparticle Production (IWCF 1996): Nijmegen, Netherlands, June 30-July 6, 1996 (World Scientific, Singapore, 1997), p. 203.

[25] Z.-G. Si, Q. Wang, and Q.-B. Xie, Phys. Lett. B 401, 107 (1997).

[26] B. R. Webber, in Proceedings: Summer School on Hadronic Aspects of Collider Physics, Zuoz, Switzerland, Aug. 23-31, 1994 (Summer School on Hadronic Aspects of Collider Physics, Zuoz, 1994), p. 49.

[27] T. Han, Z. Si, K. M. Zurek, and M. J. Strassler, J. High Energy Phys. 07 (2008) 008.

[28] R. Aaij et al. (LHCb Collaboration), J. High Energy Phys. 01 (2013) 090.

[29] P. Ilten, Y. Soreq, J. Thaler, M. Williams, and W. Xue, Phys. Rev. Lett. 116, 251803 (2016).

[30] Z. Chacko, H.-S. Goh, and R. Harnik, Phys. Rev. Lett. 96, 231802 (2006).

[31] The ATLAS collaboration, Tech. Rep. ATLAS-CONF2016-075, CERN, Geneva, 2016, https://cds.cern.ch/ record/2206245.

[32] V. Khachatryan et al. (CMS Collaboration), Phys. Rev. D 91, 052012 (2015).

[33] The ATLAS collaboration, Tech. Rep. ATL-PHYS-PUB2015-023, CERN, Geneva, 2015, http://cds.cern.ch/record/ 2037700.

[34] V. Khachatryan et al. (CMS Collaboration), J. Instrum. 10, P02006 (2015).

[35] A. M. Sirunyan et al. (CMS Collaboration), J. High Energy Phys. 07 (2017) 014.

[36] Y. Cui and B. Shuve, J. High Energy Phys. 02 (2015) 049.

[37] R. Aaij et al. (LHCb Collaboration), J. Instrum. 10, P06013 (2015).

[38] Y. Gershtein, Phys. Rev. D 96, 035027 (2017). 